\title{
Investigation of Size Effect in Absorption Spectra of Silver and Gold Nanoparticles
}

\author{
Mohammad Javad Azarm, Alireza Keshavarz, and Gholamreza Honarasa \\ Department of Physics, Shiraz University of Technology, Shiraz, Iran \\ Corresponding author email: $\underline{\text { m.azarm@sutech.ac.ir }}$ \\ Regular paper: Received: Jan. 18, 2021, Revised: May. 21, 2021, Accepted: Aug. 24, 2021, \\ Available Online: Aug. 26, 2021, DOI: 10.52547/ijop.15.1.35
}

\begin{abstract}
The absorption cross-section of gold and silver nanoparticles has been demonstrated in confined wavelength spectra based on Mie's theory. For this purpose, the numerical study performed with COMSOL for defined particle size to clarify absorption spectra and final results have been compared with experimental data to express the absorption peak occurs in higher wavelength for large particle size which is in around 530 nanometers for gold and 400 nanometer for silver particles. These results show that particle size affects directly on absorption spectra of metallic nanoparticles.
\end{abstract}

KEYWORDS: Absorption cross section, Au and $\mathrm{Ag}$ nanoparticles, Mie theory, plasmonic effects.

\section{INTRODUCTION}

For Metal Nano-Particles (MNPs) capturing, manipulating, and concentrating light on a subwavelength scale is the cause of being attractive for chemists, physicists, material scientists, and even biologists [1]. All these remarkable properties are attributed to surface plasmon excitations at the interface of a conductor and an insulator as the result of interaction with an electromagnetic wave [2]. The coupling between light and surface plasmons in nanostructures leads to novel phenomena such as transport and storage of the energy [3]. The observation of enhancement in transporting or saving energy on metallic nanoparticle surfaces depends on incident light frequency [4], surrounding medium [5], size, and shape of sub-wavelength nanoparticles [6].
Metal nanoparticles mostly Gold and Silver are structured and characterized on the nanometer scale to introduce MNPs as a prominent candidate for use as part of integrated photonic devices for instance plasmonic lasers [7], optical modulators, sensors [8], and photovoltaic devices [9]. In this regard, the efficiency enhancement of photonic devices depends on the appropriate selection of size and shape of MNPs [10]. The investigation of absorption and transition from MNPs refers to the beginning of the 19century when Mie first developed his classical electrodynamic model for metallic nanospheres; accordingly, the Mie theory analytically solved the problem of interaction between the planar electromagnetic wave and metal nanoparticle classically which leads scattering and absorption cross sections [11].

In recent years, properties of nano-scale particles are being calculated with numerical methods such as time and frequency domain methods. The assumption of frequency domain solutions like finite element method (FEM) and discrete dipole approximation (DDA) is the harmonic time evolution of the electromagnetic field [12], on the contrary, finite difference time domain method (FDTD) time-domain allows to simulate transition of electromagnetic wave [13]. Recent researches allow us to compute the plasmonic effects of spherical nanoparticles in a wide range of size in various models from classical electrodynamic to quantum mechanics methods [14]. One of the most effective semiclassical methods is Random Phase Approximation (RPA), albeit it does not 
support the solution for large particles. RPA as a semi-classical method explains the interactions of light and small metal nanoparticles [15].

In the present paper with the use of the classical Mie approach and FEM calculation employing COMSOL Multiphysics the absorption crosssection spectra of Gold (Au) and Silver (Ag) nanospheres will be demonstrated and compared with experimental results which are obtained with the chemical reduction method.

\section{THEORETICAL MODEL}

\section{A. Numerical modeling}

Mie approach is used to have a survey on plasmon resonance in MNPs, bases on an analytical solution on Maxwell equation for incident planar wave scattered and absorbed by metallic nanoparticles [16]. The weak side of this approach consists of dielectric function which is usually modeled with experimental data, although the same holds to other methods of solution of the Maxwell equation. Commonly used methods are utilizing the finite element solution method of Maxwell equations both in space and time domains, implemented in COMSOL. On the plus side, Mie and numerical FEM approaches provide the possibility of an accurate solution for incident light scattering, reflection, and absorption, closely related to real processes [17].

The numerical study of plasmon excitations in gold and silver nanoparticles have been performed in the framework of COMSOL Multiphysics 5.5 with Wave Optics module. This module allows us to solve the propagation effects of electromagnetic waves in surrounding media like a dielectric. This framework allows us to solve complicated problems without building a model from the ground up. Moreover, the framework of COMSOL was validated by comparison with the Mie Theory. Nevertheless, COMSOL has been employed mostly in simple and complicated models in 3D, 2D, and 1D geometry due to its flexibility versus shape and material set-up. In the way of simulating plasmon resonant effects, the dielectric function is needed so it has been obtained from experimental results of Johnson and Christy [18].

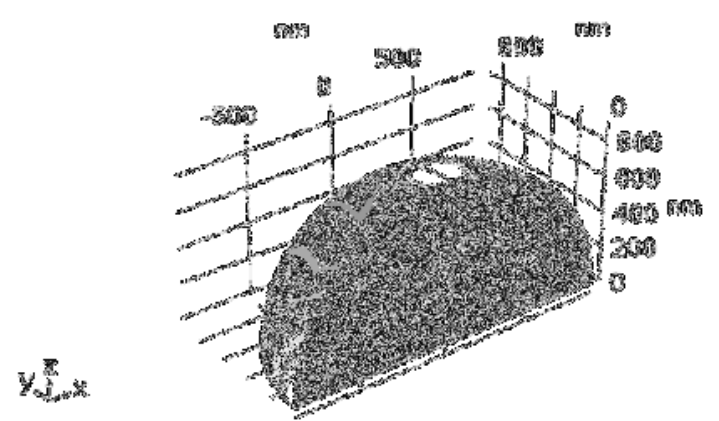

Fig. 1. Schematic of Spherical nanoparticle surrounding by dielectric and PML layers.

The simulation model was built in three domains. Fig. 1 Shows the metallic nanoparticles surrounding with dielectric media and Perfectly Matched Layer (PML), which quenches back-reflections from external surrounding boundaries. Thickness of PML layer was selected suitably to incident wavelength. built-in free meshing COMSOL algorithms help us to discretize our simulation. In this simulation tetrahedral finite elements in the area of the nano-particle and surrounding media, triangular meshing for the boundary of PML, and sweep mesh to divide PML layer into 5 spherical layers. Finally, with the use of parametric sweep, the extinction cross-section was calculated for the wavelength range around the visible area as a sum of absorption and Scattering formula. But numerically the assumption of Quasi-static conditions for particles, much smaller than the wavelength, leads to solving Laplace's equation in the spherical coordinate system. The boundary conditions bring the solution to the end by having a Poynting vector, at the end integration on the surface of nanoparticle the amount of energy will be obtained as below:

$$
\begin{aligned}
& C_{s c a}=\frac{4}{\sqrt{\varepsilon_{0} / E_{0}^{2} \mu_{0}}} \int_{V} S . \hat{n} d \Omega, \\
& C_{a b s}=\frac{4}{\sqrt{\varepsilon_{0} / E_{0}^{2} \mu_{0}}} \int_{a} U_{a} d V, \\
& C_{e x t}=C_{a b s}+C_{s c a},
\end{aligned}
$$


where $S$ is the Poynting vector, $v$ is the boundary between PML and the surrounding medium, $\hat{n}$ is the vector normal to the $v$ surface, $E_{0}$ is the amplitude of the electric component of the incident electromagnetic wave, $U_{a}$ indicates Ohmic heat losses in nanoparticle and $V$ is the nanoparticles volume.

\section{EXPERIMENTAL PROCEDURE}

\section{B. Materials and Preparation}

Hydrogen Tetrachloroauric (III) trihydrate ( $\mathrm{HAuCl} \cdot 3 \mathrm{H} 2 \mathrm{O})$, poly vinylpyrrolidone (PVP), silver nitrate $\left(\mathrm{AgNO}_{3}\right)$, and Trisodium citrate $\left(\mathrm{Na}_{3} \mathrm{C}_{6} \mathrm{H}_{5} \mathrm{O}_{7}\right)$ were purchased from Merck and $\mathrm{N}_{2}$ gas was provided to obtain Stable solutions.

It is well known that there exist different methods for the synthesis of gold nanoparticles, [19] and the citrate reduction is one of the most commonly used ways, where the stable and dispersed gold nanoparticles could be readily obtained and the size of gold nanoparticles could be easily controlled [20].

For preparation $\mathrm{Au}$ nanoparticles help of chemical reduction, the Hydrogen Tetrachloroauric dispersed in deionized water with the assistance of ultrasonic methods, the mixed solution was heated and stirred on a hot plate stirrer for $10 \mathrm{~min}$ to bring the solution just to boil with stirring subsequently, Pre-prepared solution of $(0.5 \mathrm{M})$ trisodium citrate was added slowly to solution. The remarkable point of adding reduction solution is the more we add, the smaller particles we attain [21]. So different volumes of trisodium citrate were added to the solution and three kinds of gold particles size have been achieved. For the synthesis of Ag nanoparticles, a sustainable amount of (PVP) was dissolved in water at room temperature and then silver nitrate was introduced into the mixture then the sealed bottle heated. With the heating time, the shape and size of Ag nanoparticles could be controlled. And the last step of preparation was bubbling pure nitrogen in solutions for about $20 \mathrm{~min}$ to remove oxygen. Characterization Ultraviolet-visible (UV-vis) absorbance spectroscopy measurement The UV spectra of the prepared silver or gold colloids were recorded. Deionized water was used as a reference.

\section{RESULTS AND DISCUSSION}

\section{Lab Results}

The addition of different amounts of trisodium citrate on the boiling solution will result from gold nanoparticles in a different range of sizes from 40 to 140 nanometer. Meanwhile, silver nanoparticles were obtained from various heating times. Eventually, the experimental section ends with results of absorption spectra of gold and silver nanoparticles of different sizes to obtain plasmonic absorption peak area in the visible range. Figure 2. provides the area of absorption peak for MNPs.
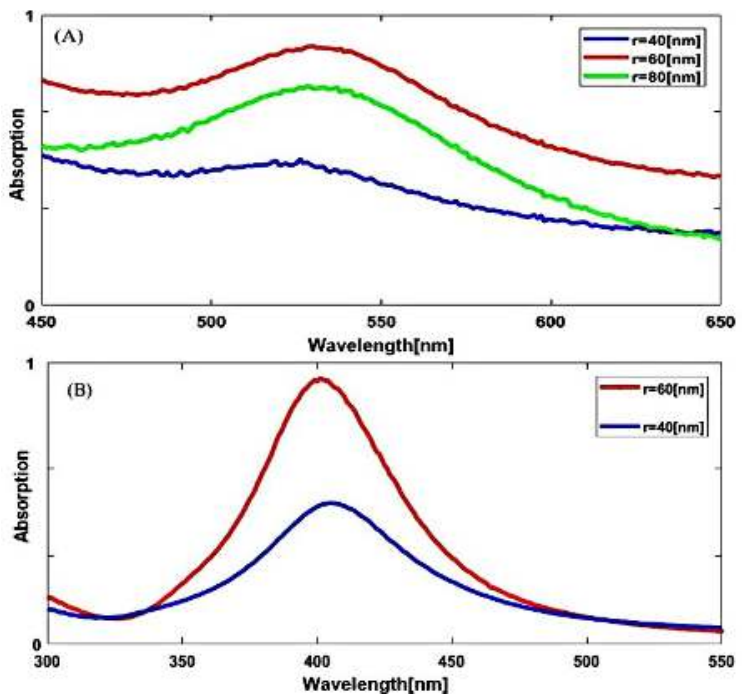

Fig. 2. Absorption spectra for (A) gold and (B) silver nanoparticles was achevied experimentally by UVvisible spectrometer.

\section{Simulation Results}

In our optical simulation, we calculate the absorption spectra of silver and gold nanoparticles to investigate the size effect of nanoparticles in plasmonic resonant. The absorption spectra were calculated for $\mathrm{Au}$ and Ag nanospheres surrounded by water $(\varepsilon=1.77)$. There is a comparison of COMSOL results (points) with Mie theory (lines) for various sizes of nanospheres of these two metals. The size range of Au nanoparticles is from $40 \mathrm{~nm}$ to $140 \mathrm{~nm}$ and silver from $20 \mathrm{~nm}$ to $60 \mathrm{~nm}$. The best agreement of Mie theory and COMSOL results corresponds to the same dielectric function 
assumed for both fully equivalent solutions of the Maxwell equation. Figure 3. shows the calculation results of absorption spectra for various sizes of gold and silver nanoparticles which were assumed spherical.
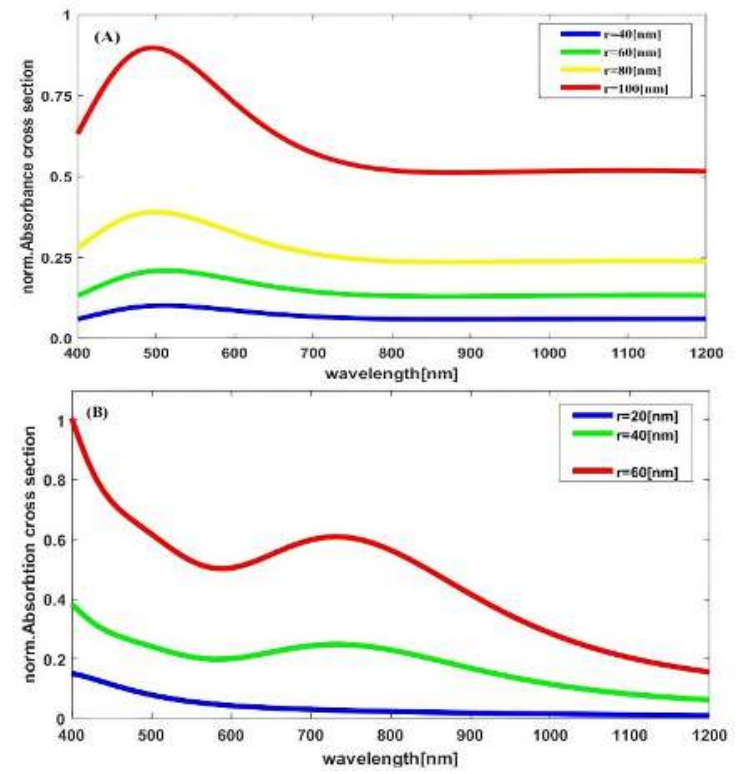

Fig. 3. Normalized absorption cross section is calculated by COMSOL for single (A) gold and (B) silver nanoparticle surrounded by water (same dielectric function assumed) Enhancement of absorption occurred for larger particles.

Moreover, most common simulation models evaluate maximum peak for $\mathrm{Au}$ and $\mathrm{Ag}$ nanoparticles around 530 and $400 \mathrm{~nm}$ Respectively and the shift in absorption spectra for larger nanoparticles had been reported in almost all simulation models furthermore, obtained results in both simulation and experimental section well coincide with other studies results.

\section{CONCLUSION}

In this paper, the red shift of plasmon resonance in metallic nanoparticles has been studied to indicate the shift, strongly for larger particles, particularly for gold and silver nanoparticles. Over and above that, both experimental and theoretical studies demonstrated surface plasmon's dependence on the size of MNPs. Fig. 4 provides the absorption peak which rose gradually for larger gold and silver water soluble particles, also these peaks occurred in higher wavelength. Dots express experimental data and lines show the simulation results. The red shift for larger particles is proved by the comparison of experimental and numerical results. In the experimental section, gold and silver nanoparticles are synthesized by chemical reduction method and characterized with UV-Visible spectrometer and absorption spectra obtained in the visible region. Meanwhile, the spectra of absorption of MNPs for various sizes have been achieved numerically by simulating a single particle in dielectric media. Finally, both investigations introduced redshift for larger particles. Accordingly, using larger gold and silver nanoparticles in integrated photonic devices will bring us efficiency enhancement.

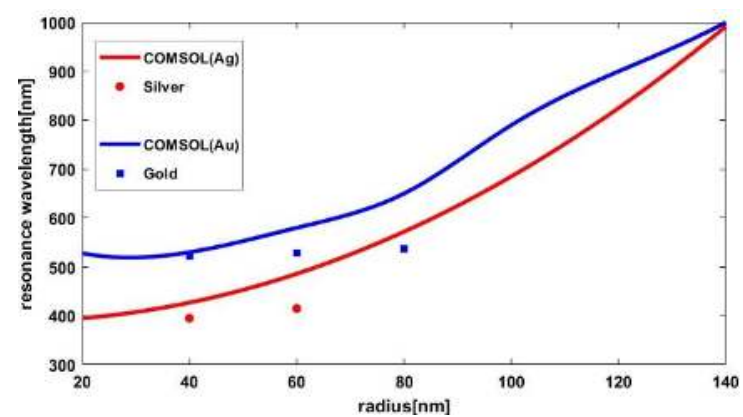

Fig. 4. Comparison of COMSOL (lines) and experimental data(dots) plasmon resonance redshift driven by gold and silver larger nanospheres.

\section{REFERENCES}

[1] Q.A. Pankhurst, J. Connolly, S.K. Jones, and J. Dobson, "Applications of magnetic nanoparticles in biomedicine," J. Phys. D: Appl. Phys. Vol. 36, pp. 220301 (1-2), 2003.

[2] E.T. Bryant and W. Garnett, "Plasmonic Properties of Metallic Nanoparticles: The Effects of Size Quantization," Nano Lett. Vol. 12, pp. 429-434, 2012.

[3] Z. Wang, P. Tao, Y. Liu, H. Xu, Q. Ye, and H. $\mathrm{Hu}$, "Rapid charging of thermal energy storage materials through plasmonic heating," Sci. Rep. Vol. 4, pp. 1-8, 2014.

[4] J.J. Mock, M. Barbic, D.R. Smith, D.A. Schultz, and S. Sc, "Shape effects in plasmon resonance of individual colloidal silver nanoparticles," J. Chem. Phys. Vol. 116, pp. 6755-6759, 2002.

[5] C. Noguez, "Surface plasmons on metal nanoparticles: the influence of shape and 
physical environment," J. Phys. Chem. C, Vol. 111, pp. 3806-3819, 2007.

[6] S. Dickreuter, J. Gleixner, A. Kolloch, J. Boneberg, E. Scheer, and P. Leiderer, "Mapping of plasmonic resonances in nanotriangles," Beilstein J. Nanotechnol, Vol. 4, pp. 588-602, 2013.

[7] R.F. Oulton, "Surface plasmon lasers: sources of nanoscopic light," Mater. Today, Vol. 15, pp. 26-34, 2012.

[8] J.A. Schuller, E.S. Barnard, W. Cai, Y.C. Jun, J.S. White, and M.L. Brongersma, "Plasmonics for extreme light concentration and manipulation," Nat. Mater, Vol. 9, pp. 193-204, 2010.

[9] H.A. Atwater and A. Polman, "Plasmonics for improved photovoltaic devices," Materials for sustainable energy: a collection of peerreviewed research and review articles from Nature Publishing Group, Vol. 9, pp. 1-11, 2011.

[10]K. Catchpole and A. Polman, "Design principles for particle plasmon enhanced solar cells," Appl. Phys. Lett. Vol. 93, pp. 191113 (13), 2008.

[11]G. Mie, "Beiträge zur Optik trüber Medien, speziell kolloidaler Metallösungen," Ann. Phys. (Berl.), Vol. 330, pp. 377-445, 1908.

[12]D. Abajo, F. Garcia, and A. Howie, "Retarded field calculation of electron energy loss in inhomogeneous dielectrics," Phys. Rev. B, Vol. 65, pp. 115418 (1-17), 2002.

[13] J.M. Montgomery, L. Tae-Woo, and S.K. Gray, "Theory and modeling of light interactions with metallic nanostructures," J. Phys.: Condens. Matter, Vol. 20, pp. 323201 (1-11), 2008.

[14]K. Kluczyk and W. Jacak, "Damping-induced size effect in surface plasmon resonance in metallic nano-particles: Comparison of RPA microscopic model with numerical finite element simulation (COMSOL) and Mie approach,” J. Quant. Spectrosc. Radiat.Vol. 168, pp. 78-88, 2016.

[15] J. Jacak, J. Krasnyj, W. Jacak, R. Gonczarek, and A. Chepok, "Surface and volume plasmons in metallic nanospheres in a semiclassical RPA-type approach: Near-field coupling of surface plasmons with the semiconductor substrate," Phys. Rev. B, Vol. 82, pp. 035418 (1-19), 2010.
[16] K. Kolwas, A. Derkachova, and M. Shopa, "Size characteristics of surface plasmons and their manifestation in scattering properties of metal particles," J. Quantitative Spectroscopy Radiative Transfer, Vol. 110, pp. 14-16, 2009.

[17]C.F. Bohren and D.R. Huffman, Absorption and scattering of light by small particles, John Wiley \& Sons, 2008.

[18] P.B. Johnson and R. Christy, "Optical constants of the noble metals," Physic. Rev. B, Vol. 6, p. 4379 (1-6), 1972.

[19] C.S. Love, C. Victor, D.K. Smith, and K. Wilson, "Synthesis of gold nanoparticles within a supramolecular gel-phase network," Chem. Commun. Vol. 15, pp. 1971-1973, 2005.

[20]G. Frens, "Controlled nucleation for the regulation of the particle size in monodisperse gold suspensions," Nat. Phys. Sci. Vol. 241, pp. 20-22, 1973.

[21] R. Zhang and X. Wang, "One step synthesis of multiwalled carbon nanotube/gold nanocomposites for enhancing electrochemical response," Chem. Mater. Vol. 19, pp. 976-978, 2007.

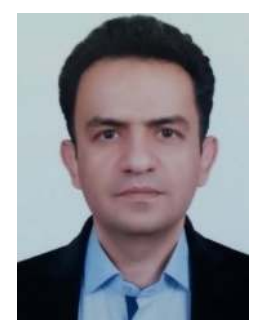

Alireza Keshavarz received his B.S. degree in Atomic Physics from Shiraz University, M.S. degree in Atomic and Molecular Physics from Kerman University, and Ph.D. degree in Physics (laser and optics) from Shiraz University. He joined the Shiraz University of Technology in 2004. He promoted to associate professor in 2012 and professor in 2016. He is interested in scientific research areas of photonics, nonlinear optics and laser, and nanotechnology. At the present, he has many M.S. and Ph.D. students who are actively involved in these fields. 


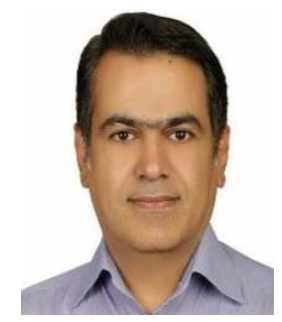

Gholamreza Honarasa was born in Iran at 1977. He received his B.S. degree and M.S. degree in Atomic and molecular Physics from Shiraz University in 2000 and 2003, respectively. He received his Ph.D. from Yazd University entitled "The study of some nonclassical features of solvable quantum systems and quantum solitons in nonlinear medium" in 2012. He is now associate professor at Shiraz University of Technology. He has published more than 25 papers and 1 book. His current research interests include laser, wave propagation and quantum optics. He is a member of Physics Society of Iran and Optics and Photonics Society of Iran.

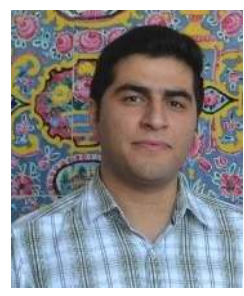

Mohamadjavad Azarm received his B.S. degree in physics from University of Isfahan, Isfahan, Iran in 2018 and M.S. degree in photonics from Shiraz University of Technology, Shiraz, Iran in 2021. His current research interests include nano-photonics, Plasmonic, laser and solar cells. 